\title{
Regularized derivatives in a 2-dimensional model of self-interacting fields with singular data
}

\author{
Günther Hörmann and Michael Kunzinger
}

\begin{abstract}
The coupled Maxwell-Lorentz system describes feed-back action of electromagnetic fields in classical electrodynamics. When applied to point-charge sources (viewed as limiting cases of charged fluids) the resulting nonlinear weakly hyperbolic system lies beyond the scope of classical distribution theory. Using regularized derivatives in the framework of Colombeau algebras of generalized functions we analyze a two-dimensional analogue of the Maxwell-Lorentz system. After establishing existence and uniqueness of solutions in this setting we derive some results on distributional limits of solutions with $\delta$-like initial values.
\end{abstract}

Keywords: Regularized derivatives, Colombeau algebras, self-interaction, weakly hyperbolic, Maxwell-Lorentz

AMS subject classification: Primary 35Dxx, 46F10, secondary 35Lxx, 78A25

\section{Introduction}

In recent years there has been considerable development in the application of nonlinear theories of generalized functions to problems in physical field theory, most successfully in general relativity (cf. [1], [14, [15], [17]) but also starting in electrodynamics (see [10]). Due to the nonlinearities inherent in the coupling of fields and equations of motion methods from classical distribution theory soon turn out to be insufficient for treating such problems. On the other hand, the theory of algebras of generalized functions in the sense of J.F. Colombeau offers the possibility of applying large classes of nonlinear operations to distributional objects.

We shortly recall the principal setup of electrodynamic field theory. Configuration space is modeled by 4-dimensional Minkowski space. The basic physical quantities are represented by the charge- and current density $J_{0}, J$, the electric field $E$, and the magnetic field $B$. Maxwell's field equations are

$$
\begin{aligned}
& \text { (H1) } \operatorname{div} B=0 \quad \text { (H2) } \partial_{t} B+\operatorname{curl} E=0 \\
& \text { (I1) } \quad \operatorname{div} E=J_{0} \quad \text { (I2) } \partial_{t} E-\operatorname{curl} B=-J
\end{aligned}
$$

with initial conditions

$$
\left.E\right|_{t=0}=\left.E_{0} \quad B\right|_{t=0}=B_{0}
$$

Institut für Mathematik, Universität Wien, A-1090 Wien, Austria

Email: guenther.hoermann@univie.ac.at, michael.kunzinger@univie.ac.at 
These equations imply the following well-known compatibility relations

$$
\begin{gathered}
\operatorname{div} B_{0}=0, \operatorname{div} E_{0}=\left.J_{0}\right|_{t=0} \\
\partial_{t} J_{0}+\operatorname{div} J=0
\end{gathered}
$$

On the other hand $(\mathrm{C} 0)$, (C1) together with $(\mathrm{H} 2)$, (I2), and the initial conditions are sufficient to derive equations (H1), (I1) (take the divergence of equations (H2), (I2) and integrate with respect to time). So, in order to solve the complete Maxwell system it is sufficient to study the hyperbolic Cauchy problem consisting of initial data and equations (H2), (I2) while $E_{0}, B_{0}, J$, and $J_{0}$ are assumed to obey the relations (C0), (C1).

Let us now investigate the nature and role of the charge density $J_{0}$ and the current $J$. In an idealized model we will interpret these as quantities of a charged fluid (or sometimes clouds of charged dust, cf. [12]) with charge density proportional to mass density (for convenience we set this ratio equal to 1 ). In this model we write

$$
\left(J_{0}, J\right)=\rho\left(u_{0}, u\right)
$$

where $\rho$ represents charge density distribution in space-time and $\left(u_{0}, u\right)$ is the 4 -velocity field of the fluid, normalized with respect to the Minkowski metric by

$$
u_{0}^{2}-|u|^{2}=1
$$

which is plausible as the 4-velocity is always assumed to be time-like.

If we study the feed-back action of the electromagnetic field on the charged fluid the equations of motion for $u$ are determined by the Lorentz force according to (cf. [12])

$$
\partial_{t} u+\sum_{j=1}^{3} \frac{u_{j}}{\sqrt{1+|u|^{2}}} \partial_{j} u=E+\frac{u}{\sqrt{1+|u|^{2}}} \times B
$$

Observe that the above formulae involve products of the basic physical quantities (resp. of their coefficients). These quantities can be singular if for example jump discontinuities of certain conductor-dielectrica configurations are to be modeled or if point charges are studied.

For instance, if one is interested in the equations of motion of charged particles resulting from the electromagnetic field they generate then $\rho$-and consequently $E$ and $B$-involve strong singularities corresponding to concentration of charge on the particles' world line.

Therefore in considering the realistic problem of self-interaction we have to deal with nonlinear differential equations involving singular functions and products and compositions thereof.

Even worse, the equations of motion cannot be written down consistently in the framework of classical distribution theory. Therefore, often physical motivations are given for renormalization and adding corrective terms. Unfortunately these "tricks" give rise to strange behaviour of solutions to the resulting equations (e.g. the Lorentz-Dirac equation). Critical discussions of the emerging inconsistencies and the resulting unphysical 
solutions ("run-away-solution", "preaccelaration", ...) can be found in the books [16] and [12.

The aim of this paper is to analyze the mathematical structure of the coupled MaxwellLorentz system by means of a low dimensional "toy model" which displays some of the main features of the general problem. The plan of exposition is as follows: In section 2 we develop a $(1+1)$-dimensional model of self-interacting charges which will provide the basis of our further investigations. As the model equations are nonlinear, of evolution type and involve singular initial data we use the formalism of regularized derivatives in Colombeau algebras of generalized functions (cf. [7]). Section 3 presents the main existence and uniqueness result in this framework. Finally, in section 4 we consider singular initial data (point charges). We illustrate the influence of the regularization procedure on the existence of distributional limits of the unique solutions established above.

\section{A $(1+1)$-dimensional model of self-interacting charges}

We try to mimic the purely mathematical structure of Maxwell equations with only one space dimension, i.e. in a two-dimensional Minkowski space $M$ with Lorentz metric in coordinates $(t, x)$ equal to $d t^{2}-d x^{2}$. The field quantities (electric and magnetic) are scalar functions on $M$ and will be denoted by $E$ and $B$. Further "physical" quantities are represented by the scalar charge density $\rho$ and the relativistic (normalized) velocity field $\left(\sqrt{1+u^{2}}, u\right)$ of a "fluid" consisting of a collection of (strictly time-like world lines of) charged particles. Therefore $\rho\left(\sqrt{1+u^{2}}, u\right)$ represents the relativistic current of the fluid. Interpreting divergence as simple $x$-derivative and removing curl terms in the original Maxwell equations this produces the electromagnetic fields according to

$$
\begin{aligned}
\partial_{x} B=0 & \partial_{t} B=0 \\
\partial_{t} E=-\rho u & \partial_{x} E=\rho \sqrt{1+u^{2}} .
\end{aligned}
$$

Therefore we may set $B$ equal to some constant $B_{0}$. The second line defines a gradient condition on $E$ which is solvable if and only if the following (relativistic) charge conservation holds

$$
\partial_{t}\left(\rho \sqrt{1+u^{2}}\right)+\partial_{x}(\rho u)=0 .
$$

In order to recover an evolution type system we simply combine the equations for $E$ into one by adding them.

Finally the feed-back action of the field on the charged particles of the fluid is modeled by mimicking the structure of the Lorentz force law:

$$
\partial_{t} u+\frac{u}{\sqrt{1+u^{2}}} \partial_{x} u=E+\frac{u}{\sqrt{1+u^{2}}} B
$$

The left hand side evaluated along a world line $z$ with $\dot{z}(s)=u(z(s))$ gives $\ddot{z}(s)$, i.e. an actual acceleration.

Again, the above formulae involve products (and even more complicated nonlinear operations) of the basic physical quantities. By the transformation $\rho \mapsto \sigma=\rho \sqrt{1+u^{2}}$ we can 
rewrite the system of equations in evolution form

$$
\partial_{t}\left(\begin{array}{c}
E \\
u \\
\sigma
\end{array}\right)+\left(\begin{array}{ccc}
1 & 0 & 0 \\
0 & \frac{u}{\sqrt{1+u^{2}}} & 0 \\
0 & \frac{u}{\left(1+u^{2}\right)^{3 / 2}} & \frac{u}{\sqrt{1+u^{2}}}
\end{array}\right) \partial_{x}\left(\begin{array}{c}
E \\
u \\
\sigma
\end{array}\right)=\left(\begin{array}{c}
\sigma-\sigma \frac{u}{\sqrt{1+u^{2}}} \\
E+\frac{u}{\sqrt{1+u^{2}}} B_{0} \\
0
\end{array}\right)
$$

Recall that $E, u$, and $\sigma$ are real valued functions of the variables $(t, x)$ and $B_{0}$ is a real constant. We study the Cauchy problem for system (2.3) with initial data

$$
\left(\begin{array}{l}
E \\
u \\
\sigma
\end{array}\right)=\left(\begin{array}{l}
E_{0} \\
u_{0} \\
\sigma_{0}
\end{array}\right) \quad \text { when } t=0
$$

System (2.3) is quasi-linear hyperbolic but not strictly hyperbolic. Also, its coefficients are not uniformly bounded. Furthermore, the main application we have in mind is to model self-interaction of charged particles (approximated by fluid clusters shrinking to isolated points) which qualitatively corresponds to $\sigma_{0}$ being proportional to a delta distribution. In order to address such questions we need a mathematical theory which is capable of describing singular objects and allows for an unrestricted application of both differentiation and of a sufficiently large class of nonlinear operations. Differential algebras of generalized functions containing the space of distributions as a subspace and the space of smooth functions as a faithful subalgebra and providing the above-mentioned tools were introduced by J.F. Colombeau in [2] (see also [3], [11]). We will therefore work in this framework.

Using the relation $\partial_{x}\left(1+u^{2}\right)^{1 / 2}=u\left(1+u^{2}\right)^{-1 / 2} \partial_{x} u$ and denoting $a(y)=y\left(1+y^{2}\right)^{-1 / 2}$ we can rewrite the above system in the more compact form

$$
\begin{aligned}
\partial_{t} E+\partial_{x} E & =\sigma(1-a(u)) \\
\partial_{t} u+\partial_{x}\left(1+u^{2}\right)^{\frac{1}{2}} & =E+B_{0} a(u) \\
\partial_{t} \sigma+\partial_{x}(\sigma a(u)) & =0 .
\end{aligned}
$$

This form of the equations will be our starting point for the method of regularized derivatives which is the subject of the following section.

\section{Regularized derivatives - existence and uniqueness of solutions in Colombeau algebras}

We will work with a variant of Colombeau algebras denoted by $\mathcal{G}_{\mathrm{s}, \mathrm{g}}(\bar{\Omega})(\Omega$ an open subset of $\mathbb{R}^{n}$ ) which is a simplified version with representatives globally bounded and smooth (cf. [7]): denote by $\mathcal{D}_{\mathrm{L}^{\infty}}(\bar{\Omega})$ the algebra of restrictions of smooth functions on $\mathbb{R}^{n}$ to $\bar{\Omega}$ which have all derivatives in $\mathrm{L}^{\infty}\left(\mathbb{R}^{n}\right)$; then within the algebra $\mathcal{E}_{\mathrm{s}, \mathrm{g}}(\bar{\Omega})=\mathcal{D}_{\mathrm{L}^{\infty}}(\bar{\Omega})^{(0, \infty)}$ we define

$$
\begin{aligned}
\mathcal{E}_{\mathrm{M}, \mathrm{s}, \mathrm{g}}(\bar{\Omega}) & =\left\{\left(u_{\varepsilon}\right)_{\varepsilon} \in \mathcal{E}_{\mathrm{s}, \mathrm{g}}(\bar{\Omega}) \mid \forall \alpha \in \mathbb{N}_{0}^{n} \exists p>0:\left\|\partial^{\alpha} u_{\varepsilon}\right\|_{\mathrm{L}^{\infty}(\bar{\Omega})}=O\left(\varepsilon^{-p}\right)(\varepsilon \rightarrow 0)\right\} \\
\mathcal{N}_{\mathrm{s}, \mathrm{g}}(\bar{\Omega}) & =\left\{\left(u_{\varepsilon}\right)_{\varepsilon} \in \mathcal{E}_{\mathrm{s}, \mathrm{g}}(\bar{\Omega}) \mid \forall \alpha \in \mathbb{N}_{0}^{n} \forall q>0:\left\|\partial^{\alpha} u_{\varepsilon}\right\|_{\mathrm{L}^{\infty}(\bar{\Omega})}=O\left(\varepsilon^{q}\right)(\varepsilon \rightarrow 0)\right\} ;
\end{aligned}
$$


then $\mathcal{G}_{\mathrm{s}, \mathrm{g}}(\bar{\Omega})$ is the quotient algebra of $\mathcal{E}_{\mathrm{M}, \mathrm{s}, \mathrm{g}}(\bar{\Omega})$ modulo $\mathcal{N}_{\mathrm{s}, \mathrm{g}}(\bar{\Omega})$. In our application we will have $\Omega=(-T, T) \times \mathbb{R}$ for $T>0$ arbitrary.

The notion of regularized derivative was introduced in [4]-[7]. It extends weak solution concepts in algebras of generalized functions. The idea is to not only represent singular functions by (classes of) regularizations but also to consider differentiation as a limiting case of more smooth operations, namely convolution with derivatives of delta regularizations. This means that a partial derivative $\partial_{j} U$ of the generalized function $U=\operatorname{cl}\left[\left(u_{\varepsilon}\right)_{\varepsilon}\right]$ will be replaced by $\operatorname{cl}\left[\left(\partial_{j} \psi_{\varepsilon} * u_{\varepsilon}\right)_{\varepsilon}\right]$ where $\left(\psi_{\varepsilon}\right)_{\varepsilon}$ is a delta net (in view of application to our model only first order derivatives are considered here). Thus if $U$ is associated to a distribution $T$ the regularized derivative will be associated to $\partial_{j} T$.

Regularized derivatives provide a powerful solution concept for a wide variety of partial differential equations. For example, the Cauchy problem for quasilinear evolution type systems

$$
\begin{aligned}
& \partial_{t} u(t, x)=\sum_{|\alpha| \leq m} A_{\alpha}(u(t, x)) \partial^{\alpha} u(t, x)+B(u(t, x)) \\
& u(0, x)=u_{0}(x)
\end{aligned}
$$

is uniquely solvable in this framework provided that $A_{\alpha}$ and $B$ are bounded in all derivatives ([7], Thm. 5.1).

The choice of which of the occurring derivatives are to be regularized may depend on special information about the model, the structure and form of the equations, and the type of effects one wants to observe. In our case we will systematically replace all $x$ derivatives in the model equations of the form (2.5-2.7) but leave the time derivatives unchanged in order to reflect the evolution type of the equations. Note that this is different from regularizing $x$-derivatives in (2.3).

The regularized $x$-derivative is defined by choosing a function $\varphi \in \mathcal{D}$ with integral equal to 1 on $\mathbb{R}$ - we call this a mollifier henceforth - and an increasing function $h:(0, \infty) \rightarrow(0, \infty)$ with $h(\varepsilon) \rightarrow 0$ as $\varepsilon \rightarrow 0$. Setting $\varphi_{\nu}(x)=\varphi(x / \nu) / \nu$ we have $\partial_{x}\left(\varphi_{\nu}(x)\right)=\varphi_{\nu}^{\prime}(x)=$ $\varphi^{\prime}(x / \nu) / \nu^{2}$ (by $\varphi_{\nu}^{\prime}$ we will always understand $\left.\left(\varphi_{\nu}\right)^{\prime}\right)$ and define the regularized derivative of a generalized function $U=\operatorname{cl}\left[\left(u_{\varepsilon}\right)_{\varepsilon}\right]$ by the formula

$$
\left(\tilde{\partial}_{x}\right)_{h} U=\operatorname{cl}\left[\left(\varphi_{h(\varepsilon)}^{\prime} * u_{\varepsilon}\right)_{\varepsilon}\right]
$$

The function $h$ can be used to control the speed of convergence towards the usual derivative compared to the regularization parameter $\varepsilon$. That it is well-defined and also the basic properties of the regularized derivative follow from the estimates

$$
\begin{gathered}
\left\|\partial^{\alpha} \partial_{x} \varphi_{h(\varepsilon)} * u_{\varepsilon}\right\|_{L^{\infty}} \leq\|\varphi\|_{L^{1}}\left\|\partial^{\alpha} \partial_{x} u_{\varepsilon}\right\|_{L^{\infty}} \\
\left\|\partial_{x} \varphi_{h(\varepsilon)} * u_{\varepsilon}\right\|_{L^{\infty}} \leq h(\varepsilon)^{-1}\left\|\varphi^{\prime}\right\|_{L^{1}}\left\|u_{\varepsilon}\right\|_{L^{\infty}} .
\end{gathered}
$$

Now we can state the main existence and uniqueness result for the Cauchy problem with regularized $x$-derivatives. We will only have to impose the following growth condition on $h$ : 
$\forall p \in \mathbb{N} \exists k \in \mathbb{N}$ s.t. $\exp \left(h(\varepsilon)^{-p}\right)=O\left(\varepsilon^{-k}\right) \quad(\varepsilon \rightarrow 0)$

For example, $h(\varepsilon)=C \ln (|\ln (\varepsilon)|)^{-1}$ satisfies $(*)$.

Theorem 1. Let $T>0$ and assume that $h$ satisfies $(*)$. Then given $E_{0}, u_{0}, \sigma_{0}$ in $\mathcal{G}_{\mathrm{s}, \mathrm{g}}(\mathbb{R})$ the system

$$
\begin{aligned}
\partial_{t} E+\left(\tilde{\partial}_{x}\right)_{h} E & =\sigma(1-a(u)) \\
\partial_{t} u+\left(\tilde{\partial}_{x}\right)_{h}\left(1+u^{2}\right)^{\frac{1}{2}} & =E+B_{0} a(u) \\
\partial_{t} \sigma+\left(\tilde{\partial}_{x}\right)_{h}(\sigma a(u)) & =0
\end{aligned}
$$

with initial conditions

$$
\left.E\right|_{t=0}=E_{0},\left.\quad u\right|_{t=0}=u_{0},\left.\quad \sigma\right|_{t=0}=\sigma_{0}
$$

has a unique solution $(E, u, \sigma)$ in $\mathcal{G}_{\mathrm{s}, \mathrm{g}}([-T, T] \times \mathbb{R})^{3}$.

Proof. We will proceed in two steps: First, the candidate for a Colombeau solution will be built up by nets of smooth solutions at fixed $\varepsilon$. Second, existence and uniqueness in $\mathcal{G}_{\mathrm{s}, \mathrm{g}}$ will be proved by verifying additional estimates with respect to the regularization-parameter $\varepsilon$. To begin with, we consider global solvability of the corresponding integro-differential equations where we will suppress all references to the index $\varepsilon$.

Step 1: integrating with respect to $t$ and inserting initial conditions we get an equivalent system of integral equations

$$
\begin{aligned}
\left(\begin{array}{c}
E \\
u \\
\sigma
\end{array}\right)(t, x)= & \left(\begin{array}{c}
E_{0} \\
u_{0} \\
\sigma_{0}
\end{array}\right)(x)-\int_{0}^{t}\left(\begin{array}{c}
E(\tau, .) * \varphi^{\prime} \\
\left(1+u(\tau, .)^{2}\right)^{1 / 2} * \varphi^{\prime} \\
a(u(\tau, .)) \sigma(\tau, .) * \varphi^{\prime}
\end{array}\right)(x) d \tau+ \\
& +\int_{0}^{t}\left(\begin{array}{c}
\sigma(\tau, x)(1-a(u(\tau, x))) \\
E(\tau, x)+B_{0} a(u(\tau, x)) \\
0
\end{array}\right) d \tau .
\end{aligned}
$$

Writing $V=\left(v_{1}, v_{2}, v_{3}\right)$ instead of $(E, u, \sigma)$ and denoting

$$
\|V\|_{T}=\max _{j=1,2,3} \sup _{|t| \leq T, x \in \mathbb{R}}\left|v_{j}(t, x)\right|
$$

and $V_{0}=\left(E_{0}, u_{0}, \sigma_{0}\right)$, which is globally bounded by assumption, we consider the right hand side as an operator $R$ on the set

$$
B_{T}\left(V_{0}\right)=\left\{V \in \mathrm{C}([-T, T] \times \mathbb{R})^{3} \mid\left\|V-V_{0}\right\|_{T} \leq 1\right\} .
$$

Using $\|a\|_{\mathrm{L}^{\infty}} \leq 1, \sqrt{1+s^{2}} \leq 1+s$ for $s \geq 0$, and Young's inequality for the convolutions, one derives from (3.8)

$$
\left|(R V)_{j}(t, x)-\left(V_{0}\right)_{j}(x)\right| \leq T\left\|\varphi^{\prime}\right\|_{L^{1}}\left(1+\|V\|_{T}\right)+T\left(2\|V\|_{T}+\left|B_{0}\right|\right) \quad(j=1,2,3)
$$


which shows that $R V \in B_{T}\left(V_{0}\right)$ for $T$ small enough. To see that $R$ is actually a contraction on $B_{T}\left(V_{0}\right)$ for small $T$ we can estimate the terms occurring in $\|R V-R W\|_{T}$ for $V$, $W \in B_{T}\left(V_{0}\right)$ after applying Young's inequality and/or taking sup for all integrands. First, in the integral involving convolutions the non-trivial factors are handled as follows: by the mean value theorem applied to the function $f(y)=\sqrt{1+y^{2}}$ and $\left\|f^{\prime}\right\|_{L^{\infty}}=\|a\|_{L^{\infty}} \leq 1$ we have $\sup \left|\sqrt{1+v_{2}^{2}}-\sqrt{1+w_{2}^{2}}\right| \leq\|V-W\|_{T}$; since also $a^{\prime}$ is globally bounded by 1 and by definition of $B_{T}\left(V_{0}\right)$ we further estimate

$$
\begin{array}{r}
\left|a\left(v_{2}\right) v_{3}-a\left(w_{2}\right) w_{3}\right| \leq\left|a\left(v_{2}\right)\left(v_{3}-w_{3}\right)\right|+\left|\left(a\left(v_{2}\right)-a\left(w_{2}\right)\right) w_{3}\right| \\
\leq\left|v_{3}-w_{3}\right|+\left|v_{2}-w_{3}\right|\left|w_{3}\right| \leq\|V-W\|_{T}\left(1+\left\|V_{0}\right\|_{L^{\infty}}\right)
\end{array}
$$

For dominating the sup of the integrands in the second integral we simply observe

$$
\begin{array}{r}
\left|v_{3}\left(1-a\left(v_{2}\right)\right)-w_{3}\left(1-a\left(w_{2}\right)\right)\right| \leq\left|v_{3}-w_{3}\right|+\left|a\left(v_{2}\right) v_{3}-a\left(w_{2}\right) w_{3}\right| \\
\left|v_{1}+B_{0} a\left(v_{2}\right)-w_{1}-B_{0} a\left(w_{2}\right)\right| \leq\left|v_{1}-w_{1}\right|+\left|B_{0}\right|\left|a\left(v_{2}\right)-a\left(w_{2}\right)\right|
\end{array}
$$

to get terms we already estimated above. Altogether for $T$ small, depending on $\left|B_{0}\right|$, $\left\|V_{0}\right\|_{\mathrm{L}^{\infty}}$, and $\left\|\varphi^{\prime}\right\|_{\mathrm{L}^{1}}$ the map $R: B_{T}\left(V_{0}\right) \rightarrow B_{T}\left(V_{0}\right)$ is a contraction. Therefore a solution of the fixed point equation exists for small $T$.

Assuming that $V$ and $W$ are two fixed points for $T>0$ arbitrary one can estimate their difference similar to the above except that only $\sup _{x \in \mathbb{R}}$ is to be considered inside integrals with respect to $\tau$. A standard Gronwall argument then yields global uniqueness of the solution. An a priori estimate for a solution $V$ in $[-T, T] \times \mathbb{R}$ is derived by using the fixed point formula (3.8), $\left|\left(1+s^{2}\right)^{1 / 2}-1\right| \leq|s|$, taking $\sup _{x}$ inside the integrals, and again applying Gronwall's lemma:

$$
\|V\|_{T} \leq\left(\left\|V_{0}\right\|_{\mathrm{L}^{\infty}}+T\left(\left\|\varphi^{\prime}\right\|_{\mathrm{L}^{1}}+\left|B_{0}\right|\right)\right) e^{T\left(\left\|\varphi^{\prime}\right\|_{\mathrm{L}^{1}}+1\right)}
$$

Therefore existence of a global solution follows. Clearly this solution is bounded on $[-T, T] \times \mathbb{R}$ by definition of $B_{T}\left(V_{0}\right)$.

Finally to get unique solutions in $\mathcal{D}_{\mathrm{L}^{\infty}}([-T, T] \times \mathbb{R})$ from initial data in $\mathcal{D}_{\mathrm{L}^{\infty}}(\mathbb{R})$ we proceed inductively. Set $B_{T}^{0}\left(V_{0}\right)=B_{T}\left(V_{0}\right)$ with $\|V\|_{T, 0}=\|V\|_{T}$; then for $k \in \mathbb{N}$ we define

$$
B_{T}^{k}\left(V_{0}\right)=\left\{V \in \mathrm{C}^{k}([-T, T] \times \mathbb{R})^{3} \mid\left\|V-V_{0}\right\|_{T, k} \leq 1\right\}
$$

where

$$
\|V\|_{T, k}=\|V\|_{T, k-1}+T\left(\left\|\partial_{t} V\right\|_{T, k-1}+\left\|\partial_{x} V\right\|_{T, k-1}\right) .
$$

Then estimates can be done essentially by the same arguments as above because the nonlinear combinations of unknowns remain of the same type (e.g. all derivatives of the function $a$ are again bounded and therefore application of Young's inequality, mean value theorem, and Gronwall's lemma are still possible). The weight factor $T^{k}$ for derivatives of order $k$ in the above norms serves to control inner derivatives and assures again e.g. the contraction property for small $T$. This concludes the purely classical base for the next step. 
Step 2: Existence and uniqueness of solutions in $\mathcal{G}_{\mathrm{s}, \mathrm{g}}([-T, T] \times \mathbb{R})^{3}$.

In equation (3.8) all involved functions except $\varphi$ now carry an index $\varepsilon$ and $\varphi$ is to be replaced by $\varphi_{h(\varepsilon)}$. We assume $\left(\left(E_{0 \varepsilon}, u_{0 \varepsilon}, \sigma_{0 \varepsilon}\right)\right)_{\varepsilon} \in \mathcal{E}_{\mathrm{M}, \mathrm{s}, \mathrm{g}}(\mathbb{R})^{3}$. In order to establish existence of a solution we will show that the net $\left(V_{\varepsilon}\right)=\left(\left(E_{\varepsilon}, u_{\varepsilon}, \sigma_{\varepsilon}\right)\right)_{\varepsilon}$ belongs to $\mathcal{E}_{\mathrm{M}, \mathrm{s}, \mathrm{g}}([-T, T] \times \mathbb{R})^{3}$.

From the integral equations for $\left(V_{\varepsilon}\right)$ and using Gronwall-type arguments as above we conclude

$$
\begin{aligned}
& \max _{1 \leq i \leq 3} \sup _{|t| \leq T, x \in \mathbb{R}}\left|v_{i, \varepsilon}(t, x)\right| \leq \\
& \leq C\left(\left\|V_{0, \varepsilon}\right\|_{\mathrm{L}^{\infty}}, T,\left\|\varphi^{\prime}\right\|_{\mathrm{L}^{1}}\right)\left|B_{0, \varepsilon}\right| h(\varepsilon)^{-1} \exp \left(3 T h(\varepsilon)^{-1}\left\|\varphi^{\prime}\right\|_{\mathrm{L}^{1}}\right),
\end{aligned}
$$

which (by $(*)$ ) gives the $\mathcal{E}_{\mathrm{M}}$-estimates of order 0 . The $\mathcal{E}_{\mathrm{M}^{-}}$-estimates for the $x$-derivatives of higher order follow inductively by similar arguments if in each step we write the $x$ derivative of any convolution product of $\varphi^{(m)}$ with any function $f$ as $\varphi^{(m+1)} * f$ and use $(*)$. The $\mathcal{E}_{\mathrm{M}}$-estimates for the $t$-derivatives follow directly by induction from the differential equations.

Concerning uniqueness, suppose that $W=\operatorname{cl}\left[\left(W_{\varepsilon}\right)_{\varepsilon}\right]$ is another solution in $\mathcal{G}_{\mathrm{s}, \mathrm{g}}([-T, T] \times$ $\mathbb{R})^{3}$. This means that there exists some $N=\operatorname{cl}\left[\left(N_{\varepsilon}\right)_{\varepsilon}\right] \in \mathcal{N}([-T, T] \times \mathbb{R})^{3}$ with

$$
\begin{aligned}
\partial_{t}\left(\begin{array}{c}
v_{1, \varepsilon}-w_{1, \varepsilon} \\
v_{2, \varepsilon}-w_{2, \varepsilon} \\
v_{3, \varepsilon}-w_{3, \varepsilon}
\end{array}\right)= & -\left(\begin{array}{c}
\left(v_{1, \varepsilon}-w_{1, \varepsilon}\right) \\
\left.\left[1-v_{2, \varepsilon}^{2}\right)^{\frac{1}{2}}-\left(1-w_{2, \varepsilon}^{2}\right)^{\frac{1}{2}}\right] \\
{\left[a\left(v_{2, \varepsilon}\right) v_{3, \varepsilon}-a\left(w_{2, \varepsilon}\right) w_{3, \varepsilon}\right]}
\end{array}\right) * \varphi_{h(\varepsilon)}^{\prime}+ \\
& \left(\begin{array}{c}
v_{3, \varepsilon}\left(1-a\left(v_{2, \varepsilon}\right)\right)-w_{3, \varepsilon}\left(1-a\left(v_{3, \varepsilon}\right)\right) \\
v_{1, \varepsilon}-w_{1, \varepsilon}+B_{0, \varepsilon}\left(a\left(v_{2, \varepsilon}\right)-a\left(w_{2, \varepsilon}\right)\right) \\
0
\end{array}\right)+\left(\begin{array}{c}
N_{1, \varepsilon} \\
N_{2, \varepsilon} \\
N_{3, \varepsilon}
\end{array}\right)
\end{aligned}
$$

From this by arguments similar to those used above we inductively derive $\mathcal{N}$-estimates for each derivative of $\left(V_{\varepsilon}-W_{\varepsilon}\right)_{\varepsilon}$. Thus $V=W$ in $\mathcal{G}_{\mathrm{s}, \mathrm{g}}([-T, T] \times \mathbb{R})^{3}$.

Remark 1. Having obtained a unique solution to the field equations let us reinvestigate the behaviour of point particles subjected to the velocity field $\left(\sqrt{1+u^{2}}, u\right)$, i.e. determine its integral curves in $\mathbb{R}^{2}$. Classically, this amounts to considering the system

$$
\begin{aligned}
& \dot{z}_{0}(s)=\sqrt{1+u(z(s))^{2}} \\
& \dot{z}_{1}(s)=u(z(s)) \\
& z(0)=\left(t_{0}, x_{0}\right)
\end{aligned}
$$

where $z=\left(z_{0}, z_{1}\right): I \rightarrow \mathbb{R}^{2}$ ( $I$ some interval containing 0$)$. By the first line of (3.10) we may reparametrize via $r=z_{0}(s)$, thereby obtaining the following equation for $(r, w(r))=$ $z(s)$ :

$$
\begin{aligned}
& \dot{w}(r)=a(u(r, w(r))) \\
& w\left(t_{0}\right)=x_{0}
\end{aligned}
$$


Of particular interest to us is $u$ arising as a solution of system (3.4) - (3.6) with strongly singular (e.g. $\delta$-like) initial data. In this case, (3.11) is an example of a nonlinear ODE involving generalized functions. Note that its right-hand side contains a composition of generalized functions (defined by componentwise insertion of representatives). A theory of such equations in the framework of Colombeau generalized functions has been developed by R. Hermann and M. Oberguggenberger in [8, 9]. Since all derivatives of $a$ are globally bounded and $u \in \mathcal{G}_{\mathrm{s}, \mathrm{g}}$ it follows that $\operatorname{cl}\left[\left(a \circ u_{\varepsilon}\right)_{\varepsilon}\right]$ is in $\mathcal{G}_{\mathrm{s}, \mathrm{g}}$ and therefore of $L^{\infty}$-type (see [9], Def. 2.3). Thus [9], Thm. 3.3 ensures the existence of a solution in $\mathcal{G}$ which is actually in $\mathcal{G}_{\mathrm{s}, \mathrm{g}}$ (which follows inductively from (3.11)). In particular, this allows to derive (generalized) trajectories of charged particles without having to resort to renormalization procedures altering the actual equations of motion. This may indicate a way of avoiding the inconsistencies mentioned at the end of section 1.

\section{Point charges as initial data}

As we mentioned in the introduction we will now study the situation where the initial data $\left(E_{0}, u_{0}, \sigma_{0}\right)$ are qualitatively of the form $(0,0, \delta)$. This can be modeled by choosing $\sigma_{0}$ to be the class of a strict delta net $\left(\rho^{\varepsilon}\right)_{\varepsilon}$ in $\mathcal{G}_{\mathrm{s}, \mathrm{g}}(\mathbb{R})$ in the sense of [11], Def.7.1. According to this definition $\left(\rho^{\varepsilon}\right)_{\varepsilon>0}$ is a family of test functions in $\mathcal{D}(\mathbb{R})$ such that

$$
\begin{gathered}
\text { supp } \rho^{\varepsilon} \rightarrow\{0\} \quad \text { as } \varepsilon \rightarrow 0 \\
\int \rho^{\varepsilon}(x) d x=1 \quad \text { for all } \varepsilon>0 \\
\int\left|\rho^{\varepsilon}(x)\right| d x \quad \text { is bounded independently of } \varepsilon .
\end{gathered}
$$

By the above theorem we obtain a unique solution in $\mathcal{G}_{\mathrm{s}, \mathrm{g}}([-T, T] \times \mathbb{R})^{3}$ of system (3.4 3.6) with (3.7) for appropriate scaling function $h$. However, the dependence of the definition of the regularized derivative on the mollifier $\varphi$ is essential. This was observed already in [6] and illustrated by numerical tests. Also in the context of scalar conservation laws it was shown how the choice of "left" or "right" mollifiers allows to recover the classical entropic solutions.

We are not able to give a complete description of conditions for the existence of distributional limits of solutions of the more complicated system (3.4 3.6). Nevertheless we will present some results analyzing the consequences of employing one-sided mollifiers.

Proposition 1. Let $T>0, h$ as in Thm.1, and $\left(E_{0}, u_{0}, \sigma_{0}\right)=\left(0,0, \operatorname{cl}\left[\left(\rho^{\varepsilon}\right)_{\varepsilon}\right]\right)$ with $\left(\rho^{\varepsilon}\right)_{\varepsilon}$ a strict delta net. If $\operatorname{supp} \varphi \subseteq[-\infty, 0]$ (resp. $\left.\subseteq[0, \infty]\right)$ then the unique solution $(E, u, \sigma) \in \mathcal{G}_{\mathrm{s}, \mathrm{g}}([-T, T] \times \mathbb{R})$ to $(3.4-3.6)$ with initial conditions (3.7) is supported in $[-T, T] \times(-\infty, 0](\operatorname{resp} .[-T, T] \times[0, \infty))$.

Proof. It will suffice to consider the case of a left-sided mollifier (since a similar argument applies to the case of a right-sided mollifier). Fix some $x_{0}>0$. By our assumption on the support of $\varphi$ we have

$$
\sigma_{\varepsilon}(t, x)=-\frac{1}{h(\varepsilon)} \int_{0}^{t} \int_{-1}^{0} \varphi^{\prime}(y) a\left(u_{\varepsilon}(\tau, x-h(\varepsilon) y)\right) \sigma_{\varepsilon}(\tau, x-h(\varepsilon) y) d y d \tau
$$


for $x \geq x_{0}$ and $\varepsilon$ sufficiently small (depending on $x_{0}$ ). Also, since $a$ is globally bounded

$$
\sup _{x \geq x_{0}}\left|\sigma_{\varepsilon}(t, x)\right| \leq \frac{C}{h(\varepsilon)}\left\|\varphi^{\prime}\right\|_{L^{1}} \int_{0}^{t} \sup _{x \geq x_{0}}\left|\sigma_{\varepsilon}(t, x)\right| d \tau
$$

so $\sup _{x \geq x_{0}}\left|\sigma_{\varepsilon}(\tau, x)\right|=0$ by Gronwall's inequality which (since $x_{0}$ was arbitrary) establishes the claim for $\sigma$. Then since

$$
\sup _{x \geq x_{0}}\left|E_{\varepsilon}(t, x)\right| \leq \sup _{x \geq x_{0}}\left|\sigma_{\varepsilon}(t, x)\left(1-a\left(u_{\varepsilon}(t, x)\right)\right)\right|+\frac{C}{h(\varepsilon)}\left\|\varphi^{\prime}\right\|_{L^{1}} \int_{0}^{t} \sup _{x \geq x_{0}}\left|E_{\varepsilon}(t, x)\right| d \tau,
$$

it follows that $\sup _{x \geq x_{0}}\left|E_{\varepsilon}(t, x)\right|=0$. Finally,

$$
\begin{aligned}
\partial_{x} u_{\varepsilon}(t, x)= & -\frac{1}{h(\varepsilon)} \int_{0}^{t} \int_{-1}^{0} \varphi^{\prime}(y) a\left(u_{\varepsilon}(\tau, x-h(\varepsilon) y)\right) \partial_{x} u_{\varepsilon}(\tau, x-h(\varepsilon) y) d y d \tau+ \\
& +B_{0} \int_{0}^{t} a^{\prime}\left(u_{\varepsilon}(\tau, x)\right) \partial_{x} u_{\varepsilon}(\tau, x) d \tau
\end{aligned}
$$

From this it follows that $\partial_{x} u_{\varepsilon}(t, x)=0$ for $x>0$. But then since $\left(1+u_{\varepsilon}^{2}\right)^{1 / 2} * \varphi_{h(\varepsilon)}^{\prime}=$ $\left(a\left(u_{\varepsilon}\right) \partial_{x} u_{\varepsilon}\right) * \varphi_{h(\varepsilon)}=0$ in this region we obtain

$$
\partial_{t} u_{\varepsilon}(t, x)=B_{0} a\left(u_{\varepsilon}(t, x)\right)=B_{0} \int_{0}^{t} a^{\prime}\left(u_{\varepsilon}(\tau, x)\right) \partial_{t} u_{\varepsilon}(\tau, x) d \tau .
$$

Thus also $\partial_{t} u_{\varepsilon}(t, x)=0$ and therefore $u_{\varepsilon}(t, x)=0$ for $x>0$.

Applying this result we now demonstrate how the choice of a left mollifier can destroy the intuitive expectation of field propagation along the characteristics of the original system.

Corollary 1. The (unique) solution of (3.4-3.6), (3.7) according to Prop.1 cannot be associated to a distribution in $\mathcal{D}^{\prime}\left(\mathbb{R}^{2}\right)^{3}$.

Proof. Assume that $(E, u, \sigma) \in \mathcal{D}^{\prime}(\mathbb{R})^{3}$ is the distributional limit of $\left(E_{\varepsilon}, u_{\varepsilon}, \sigma_{\varepsilon}\right)$. Then from $\partial_{t} E_{\varepsilon}+\varphi_{h(\varepsilon)}^{\prime} * E_{\varepsilon}=\sigma_{\varepsilon}\left(1-a\left(u_{\varepsilon}\right)\right)$ it follows that $\sigma_{\varepsilon} a\left(u_{\varepsilon}\right) \rightarrow \sigma-F$, where $F=\partial_{t} E+\partial_{x} E$. Also, the third line of the regularized system implies $\partial_{t} \sigma+\partial_{x}(\sigma-F)=0$, so

$$
\partial_{t}\left(\sigma-\partial_{x} E\right)+\partial_{x}\left(\sigma-\partial_{x} E\right)=0
$$

Using the coordinate transformation $T(t, x)=(x, t-x)$ it follows that $\sigma-\partial_{x} E$ is of the form $v(t-x)$ (i.e. $T^{*}(1 \otimes v)$ ) for some $v \in \mathcal{D}^{\prime}(\mathbb{R})$, hence is contained in $\mathrm{C}^{\infty}\left(\mathbb{R}, \mathcal{D}^{\prime}(\mathbb{R})\right)$. By Prop. 1 the supports of $\sigma$ and $E$ are contained in $x \leq 0$ and from the initial conditions we get $\left(\sigma-\partial_{x} E\right)(0)=\delta(x)$. Thus $\sigma-\partial_{x} E=\delta(t-x)$, which contradicts $\operatorname{supp}\left(\sigma-\partial_{x} E\right) \subseteq$ $\{x \leq 0\}$.

It remains an open question whether there are choices of the mollifier $\varphi$ which yield distributional limits of the solution for singular initial data as in Prop.1. 
Remark 2. Let us interpret equations (2.5-2.7) in the form $P(E, u, \sigma)=0$ with the operator $P: \mathrm{C}^{\infty}\left(\mathbb{R}^{2}\right)^{3} \rightarrow \mathrm{C}^{\infty}\left(\mathbb{R}^{2}\right)^{3}$ given by

$$
P(E, u, \sigma)=\left(\begin{array}{c}
\partial_{t} E+\partial_{x} E+\sigma(a(u)-1) \\
\partial_{t} u+a(u) \partial_{x} u-E-B_{0} a(u) \\
\partial_{t} \sigma-\partial_{x}(a(u) \sigma)
\end{array}\right) .
$$

We can decompose $P$ into a sum of a linear differential operator $L$ and a (purely) nonlinear part $Q$ with

$$
\begin{gathered}
L(E, u, \sigma)=\left(\begin{array}{c}
\partial_{t} E+\partial_{x} E-\sigma \\
\partial_{t} u-E \\
\partial_{t} \sigma
\end{array}\right) \\
Q(E, u, \sigma)=\left(\begin{array}{c}
a(u) \sigma \\
a(u)\left(\partial_{x} u-B_{0}\right) \\
-\partial_{x}(a(u) \sigma)
\end{array}\right) .
\end{gathered}
$$

We observe that in a linearization of $P$ (in the sense of [13, p.20) at a point $\left(t_{0}, x_{0}\right)$ with $u\left(t_{0}, x_{0}\right)=0$ all terms corresponding to $Q$ vanish and we are left exactly with $L$. Therefore, if we consider some smooth approximation of the initial data (with $q$ the constant point charge)

$$
\left(E_{0}, u_{0}, \sigma_{0}\right)=(0,0, q \delta)
$$

and a linearization of the system near $t=0$, the unique distributional solution $(E, u, \sigma) \in$ $C^{1}\left([0, T], \mathcal{D}^{\prime}(\mathbb{R})\right)^{3}$ of the Cauchy problem

$$
\left(\begin{array}{c}
\partial_{t} E+\partial_{x} E \\
\partial_{t} u \\
\partial_{t} \sigma
\end{array}\right)=\left(\begin{array}{c}
\sigma \\
E \\
0
\end{array}\right)
$$

with initial condition (4.6) may give a hint on how to construct approximate smooth solutions of the non-linear system. The solution to the linearized system is given by

$$
\left(\begin{array}{l}
E \\
u \\
\sigma
\end{array}\right)(t, x)=q \cdot\left(\begin{array}{c}
H(x)-H(x-t) \\
(t-x)(H(x)-H(x-t)) \\
\delta(x)
\end{array}\right),
$$

where $H$ denotes the Heaviside function. However, using a standard regularization of this solution (by means of a model delta net) as a candidate for such an approximate solution runs into difficulties: explicit calculations show that the blow up of the term $\sigma a(u)$ at $x=0$ is unavoidable unless regularizations with $\delta H \approx 0$ are considered.

\section{References}

[1] Clarke, C.J.S., Vickers, J.A., Wilson, J.P., Generalized functions and distributional curvature of cosmic strings, Class. Quant. Grav. 13, No.9, 2485-2498 (1996). 
[2] Colombeau, J.F., New generalized functions and multiplication of distributions, North Holland, Amsterdam, 1984.

[3] Colombeau, J.F., Elementary introduction to new generalized functions, North Holland, Amsterdam, 1985.

[4] Colombeau, J.F., Heibig, A., Oberguggenberger, M., Le probleme de Cauchy dans un espace de fonctions generalisees I, C.R. Acad. Sci., Ser I 317, No.9, 851-855 (1993).

[5] Colombeau, J.F., Heibig, A., Oberguggenberger, M., Le probleme de Cauchy dans un espace de fonctions generalisees II, C.R. Acad. Sci., Ser I 319, No.11, 1179-1183 (1994).

[6] Colombeau, J.F., Heibig, A., Generalized Solutions to Cauchy Problems, Monatsh. Math. 117, 33-49, 1994.

[7] Colombeau, J.F., Heibig, A., Oberguggenberger, M., Generalized solutions to partial differential equations of evolution type, Acta Appl. Math. 45, 115-142, 1996.

[8] Hermann, R., Oberguggenberger, M., Generalized functions, calculus of variations, and nonlinear ODEs, preprint 1997.

[9] Hermann, R., Oberguggenberger, M., Ordinary differential equations and generalized functions, to appear in M. Grosser, G. Hörmann, M. Kunzinger, M. Oberguggenberger, Nonlinear Theory of Generalized Functions, CRC-press, Boca Raton, 1999.

[10] Hörmann, G., Kunzinger, M., Nonlinearity and Self-Interaction in Physical Field Theories with Singularities, Int. Transf. Spec. Funct. Vol. 6, No. 1-4, 205-214, 1998.

[11] Oberguggenberger, M., Multiplication of distributions and applications to partial differential equations, Pitman Research Notes in Mathematics 259, Longman 1992.

[12] Parrott, S., Relativistic Electrodynamics and Differential Geometry, Springer, New York 1987.

[13] Rauch, J., Partial Differential Equations, Springer-Verlag, New York 1991.

[14] Steinbauer, R., The ultrarelativistic Reissner-Nordstrøm field in the Colombeau algebra, J. Math. Phys. 38, No.3, 1614-1622 (1997).

[15] Steinbauer, R., Geodesics and geodesic deviation for impulsive gravitational waves, J.Math.Phys. 39 (1998) 2201-2212.

[16] Thirring, W., A Course in Mathematical Physics, Volume II, Springer-Verlag, 1979.

[17] Wilson, J.P., Distributional curvature of time-dependent cosmic strings, Class. Quant. Grav., Vol. 14, 3337-3351, 1997. 